\title{
A GENERALIZATION OF A THEOREM OF DELAUNAY
}

\author{
WU-YI HSIANG \& WEN-CI YU \\ Dedicated to Professor Buchin Su on his 80 th birthday
}

\section{Introduction}

In 1841 Delaunay [1] discovered the following beautiful way of constructing rotational symmetric hypersurfaces of constant mean curvature in euclidean 3-space $\mathbf{R}^{3}$, by rolling a given conic section on a line in a plane, and rotating about that line the trace of a focus, one obtains a hypersurface of constant mean curvature in $\mathbf{R}^{3}$. Conversely, all rotational symmetric hypersurfaces of constant mean curvature in $\mathbf{R}^{3}$, except those spheres, can be constructed in this way. Combining the above classical result with a theorem of Ruh and Vilms [6] which asserts that the Gauss map of a hypersurface of constant mean curvature in $\mathbf{R}^{n}$ is automatically harmonic, one can easily use the periodicity of such construction (for ellipse and hyperbola) to obtain harmonic maps of $T^{2} \rightarrow S^{2}$. In this paper, we shall study generalized rotational hypersurfaces of constant mean curvature in higher dimensional euclidean spaces, and prove a generalization of Delaunay theorem, which again enables us to construct harmonic maps of $S^{p} \times S^{1} \rightarrow S^{p+1}$.

From the viewpoint of equivariant differential geometry, a natural generalization of the $S O(2)$ rotation on $\mathbf{R}^{3}$ is simply an orthogonal transformation group $\left(G, \mathbf{R}^{n}\right)$ with codimension-two principal orbit type. Such orthogonal transformation groups are classified in [4]. Therefore it is quite natural to study solutions of hypersurfaces in $\mathbf{R}^{n}$ with constant mean curvature, which are invariant with respect to one of such orthogonal transformation groups with codimension-two principal orbit type.

In $\S 1$ we shall reduce the above equivariant geometric problem to a much simpler ordinary differential equation defined on a suitable two-dimensional manifold, namely, the orbit space $\mathbf{R}^{n} / G$. In the special case where $G=$ $S O(n-1)$, the corresponding ordinary differential equation can be integrated explicitly in terms of hyperelliptic functions. Furthermore, it is

Received April 13, 1981. 
possible to generalize Delaunay's rolling construction to this special case, and we shall exploit such geometric construction to discuss various properties such as periodicity of the solutions (cf. $\$ 2$ and §3). In succeeding papers we shall further investigate the properties of hypersurfaces with constant mean curvature, which are invariant under other cohomogeneity-two orthogonal transformation groups.

\section{Generalized rotational hypersurfaces of constant mean curvature in $\mathbf{R}^{\boldsymbol{n}}$}

Let $G$ be a compact connected Lie group, and $\Phi$ an orthogonal representation of $G$ acting on $\mathbf{R}^{n}$ with codimension-two principal orbit type. Such orthogonal transformation groups $\left(G, \Phi, \mathbf{R}^{n}\right)$ have been classified in [4], and the final result of such a classification can be neatly described as follows: Let $L / G$ be a symmetric space of rank 2 , and $\Phi$ the isotropy representation of $G$ on the tangent space $\mathbf{R}^{n}$ of the base point. Then it follows from the definition of rank that $\mathbf{R}^{n} / G$ is of dimension two and hence it is an example of such orthogonal transformation groups. The final result of the classification of [4] simply asserts that every orthogonal transformation group $\left(G, \Phi, \mathbf{R}^{n}\right)$ with $\operatorname{dim} \mathbf{R}^{n} / G=2$ is obtained from the isotropy representation of a symmetric space of rank 2 .

As a generalization of rotational surfaces in $\mathbf{R}^{3}$, we shall call an invariant hypersurface in $\left(G, \Phi, \mathbf{R}^{n}\right)$ a generalized rotational hypersurface [with respect to the given type of cohomogeneity-two transformation $\left(G, \Phi, \mathbf{R}^{n}\right)$ ]. Hypersurfaces of constant mean curvature in $\mathbf{R}^{n}$ are locally given by a quasi-linear second order elliptic partial differential equation which is rather difficult to deal with. Therefore our first step in the study of rotational hypersurfaces of constant mean curvature will be to make effective use of the "symmetry" of such a geometric situation to reduce the above formidable analytic problem to a more manageable one. Let us recall here some basic generalities of equivariant differential geometry. Suppose $M$ is a Riemannian manifold with a given isometric transformation group $G$. Then it is rather natural to equip the orbit space $M / G$ with the following structures: (i) a generalized smooth structure on $M / G$ whose smooth functions are exactly the quotients of $G$-invariant smooth functions, namely, $C^{\infty}(M / G) \cong C^{\infty}(M)^{G}$, (ii) a generalized Riemannian metric $d s^{2}$ on $M / G$ which measures the distances between $G$-orbits, (we shall call it the orbital distance metric of $M / G$ ), (iii) a smooth function $f: M / G \rightarrow \mathbf{R}$ such that $f(\xi)=(\text { volume of } \xi)^{2}$ if $\xi$ is a principal $G$-orbit. In term of the above orbital geometric invariants of $(G, M)$, one has 
the following proposition of [3] which reduce the computation of mean curvature of an $G$-invariant submanifold $N \subset M$ to that of its image $N / G \subset$ $M / G$.

Proposition 1. Let $N$ be an invariant submanifold of $M$ with the same principal orbit type as that of $M, x$ be a point on a principal orbit $\xi \in N / G, \nu_{x}$ be an arbitrary unit normal vector of $N$ at $x$, and $\nu_{\xi}$ be its image vector at $\xi$. Then

$$
H\left(\nu_{x}\right)=H^{\prime}\left(\nu_{\xi}\right)-\frac{1}{2} \frac{d}{d \nu_{\xi}} \ln f,
$$

where $H\left(\nu_{x}\right), H^{\prime}\left(\nu_{\xi}\right)$ are the mean curvatures of $N, N / G$ in the direction of $\nu_{x}, \nu_{\xi}$ respectively, and $d / d \nu_{\xi}$ is the differentiation in the direction of $\nu_{\xi}$.

Next, let us recall the result of some explicit computations of the orbital geometric invariants of those orthogonal transformation groups on $\mathbf{R}^{n}$ of cohomogeneity two [cf. 4]:

Suppose $\left(G, \Phi, \mathbf{R}^{n}\right)$ is an orthogonal transformation group of cohomogeneity two. Then, according to the classification of [4], $\Phi$ is the isotropy representation of a rank-2 symmetric space $L / G$. It follows from the maximal tori theorem of É. Cartan (for the case of symmetric space) that (i) there exists a 2-dimensional linear subspace $\mathbf{R}^{2}$ which interesects every $G$-orbit perpendicularly, (ii) the Weyl group of $L / G, W$, acts on $\mathbf{R}^{2}$ as a group generated by reflections and $\mathbf{R}^{n} / G \cong \mathbf{R}^{2} / W$. Therefore the orbit space $\mathbf{R}^{n} / G$ can be identified with a Weyl chamber of $\left(W, \mathbf{R}^{2}\right)$, and the orbital distance metric is flat. We shall choose an orthonormal coordinate system $(x, y)$ on $\mathbf{R}^{2}$ such that the Weyl chamber of $\left(W, \mathbf{R}^{2}\right)$ is given by $y \geqslant 0$ and $x \cdot \sin \pi / d-$ $y \cos \pi / d \geqslant 0$, namely, it is a linear cone of angle $\pi / d$ as follows:

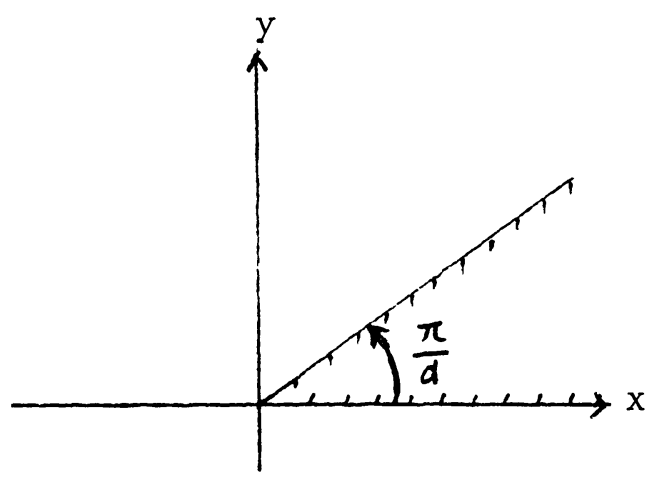

FIG. 1 
We list the needed orbital geometric invariants of those cohomogeneity-two orthogonal transformation groups $\left(G, \Phi, \mathbf{R}^{n}\right)$ as follows:

\begin{tabular}{|c|c|c|c|c|c|}
\hline$G$ & $\Phi$ & $\begin{array}{c}n= \\
\operatorname{dim} \Phi\end{array}$ & $d(W)$ & $f(\xi)=(\text { volume of } \xi)^{2}$ & $\begin{array}{c}\text { Asso. Sym. Space } \\
L / G\end{array}$ \\
\hline$S O(n-1)$ & $1+\rho_{n-1}$ & $n$ & 1 & $c y^{2 n-4}$ & $\mathbf{R}^{1} \times S^{n-1}$ \\
\hline$S O(l) \times S O(m)$ & $\rho_{l}+\rho_{m}$ & $l+m$ & 2 & $c x^{2 m-2} y^{2 l-2}$ & $S^{l} \times S^{m}$ \\
\hline$S O(3)$ & $S^{2} \rho_{3}-1$ & 5 & 3 & $c \prod_{i=0}^{2} w(3, i)^{2}$ & $S U(3) / S O(3)$ \\
\hline$S U(3)$ & Ad & 8 & 3 & $c \prod_{i=0}^{2} w(3, i)^{4}$ & $\frac{S U(3) \times S U(3)}{S U(3)}$ \\
\hline$S p(3)$ & $\Lambda^{2} \nu_{3}-1$ & 14 & 3 & $c \prod_{i=0}^{2} w(3, i)^{8}$ & $\frac{S U(6)}{S p(3)}$ \\
\hline$F_{4}$ & 1 & 26 & 3 & $c \prod_{i=0}^{2} w(3, i)^{16}$ & $\frac{E_{6}}{F_{4}}$ \\
\hline$S O(5)$ & Ad & 10 & 4 & $c x^{4} y^{4}\left(x^{2}-y^{2}\right)^{4}$ & $\frac{S O(5) \times S O(5)}{S O(5)}$ \\
\hline$S O(2) \times S O(m)$ & $\rho_{2} \otimes \rho_{m}$ & $2 m$ & 4 & $c(x y)^{2 m-4}\left(x^{2}-y^{2}\right)^{2}$ & $\frac{S O(2+m)}{S O(2) \times S O(m)}$ \\
\hline$S(U(2) \times U(m))$ & {$\left[\mu_{2} \otimes_{C} \mu_{m}\right] \mathbf{R}$} & $4 m$ & 4 & $c(x y)^{4 m-6}\left(x^{2}-y^{2}\right)^{4}$ & $\frac{S U(2+m)}{S(U(2) \times U(m))}$ \\
\hline$S p(2) \times S p(m)$ & $\nu_{2} \otimes_{H} \nu_{m}^{*}$ & $8 m$ & 4 & $c(x y)^{8 m-10}\left(x^{2}-y^{2}\right)^{8}$ & $\frac{S p(2+m)}{S p(2) \times S p(m)}$ \\
\hline$U(5)$ & {$\left[\Lambda^{2} \mu_{5}\right]_{R}$} & 20 & 4 & $c(x y)^{10}\left(x^{2}-y^{2}\right)^{8}$ & $\frac{S O(10)}{U(5)}$ \\
\hline$U(1) \times \operatorname{Spin}(10)$ & {$\left[\mu_{1} \otimes_{C} \Delta_{1}^{+}\right]_{R}$} & 32 & 4 & $c(x y)^{18}\left(x^{2}-y^{2}\right)^{12}$ & $\frac{E_{6}}{U(1) \times \operatorname{Spin}(10)}$ \\
\hline$G_{2}$ & Ad & 14 & 6 & $c \prod_{i=0}^{5} w(6, i)^{4}$ & $\frac{G_{2} \times G_{2}}{G_{2}}$ \\
\hline$S O(4)$ & $\begin{array}{ll}1 & 3 \\
0 & 0\end{array}$ & 8 & 6 & $c \prod_{i=0}^{5} w(6, i)$ & $G_{2} / S O(4)$ \\
\hline
\end{tabular}

where $\rho_{m}, \mu_{m}, \nu_{m}$ are the birth-certificate representations of $S O(m), S U(m)$ or $U(m), S p(m)$ respectively, and $w(d, i)$ is the following linear function:

$$
w(d, i)=x \sin \frac{i \pi}{d}-y \cos \frac{i \pi}{d} .
$$

Combining the above list of geometric invariants with Proposition 1, we can easily write down the corresponding ordinary differential equation satisfied by the image curve $N / G \subset \mathbf{R}^{n} / G \cong \mathbf{R}^{2} / W$ of a generalized rotational hypersurface $N$ of constant mean curvature in each of the above 14 cases. For 
example, in the first two cases, the corresponding equations are simply as follows:

$$
(G, \Phi)=\left(S O(n-1), 1+\rho_{n-1}\right):
$$

$$
\begin{aligned}
& \dot{x} \ddot{y}-\dot{y} \ddot{x}-(n-2) \frac{\dot{x}}{y}=h(\text { constant }), \\
& \quad(G, \Phi)=\left(S O(l) \times S O(m), \rho_{l}+\rho_{m}\right): \\
& \dot{x} \ddot{y}-\dot{y} \ddot{x}-(l-1) \frac{\dot{x}}{y}+(m-1) \frac{\dot{y}}{x}=h \text { (constant), }
\end{aligned}
$$

where $\dot{x}, \dot{y}, \ddot{x}, \ddot{y}$ are derivatives with respective to the euclidean arc length $d s^{2}=d x^{2}+d y^{2}$ of $\mathbf{R}^{n} / G \cong \mathbf{R}^{2} / W \subset \mathbf{R}^{2}$, and $(x(s), y(s))$ is the image curve $N / G$ of a generalized rotational hypersurface of constant mean curvature $h$.

In the first case, if one considers $y$ as a function of $x, y(x)$, then (2) is equivalent to the following:

$$
\begin{aligned}
& {\left[\frac{y^{n-2}}{\sqrt{1+y^{\prime 2}}}+\frac{h}{(n-1)} y^{n-1}\right]^{\prime}} \\
& =-y^{n-2} y^{\prime}\left[\frac{y^{\prime \prime}}{\left(1+y^{\prime 2}\right)^{3 / 2}}-\frac{(n-2)}{y\left(1+y^{\prime 2}\right)^{1 / 2}}-h\right]=0 .
\end{aligned}
$$

Therefore it is easy to integrate the above equation explicitly in terms of hyperelliptic functions, namely,

$$
\begin{aligned}
& \frac{y^{n-2}}{\sqrt{1+y^{\prime 2}}}+\frac{h}{n-1} y^{n-1}=c, \\
& x=\int\left[\left(\frac{y^{n-2}}{c-\frac{h}{(n-1)} y^{n-1}}\right]^{2}-1\right]^{-1 / 2} d y .
\end{aligned}
$$

In the beginning case of $n=3$, the above integral is an elliptical integral which is, by definition, closely related to the arc-length of an ellipse. The classical theorem of Delaunay neatly demonstrate the explicit relationship of the above geometrically arrived function $y(x)$ by a simple geometric construction, namely, by rolling an ellipse and plotting the trace of one of its focus. In the next section, we shall prove a generalization of the above theorem of Delaunay to construct higher dimensional rotational hypersurfaces of constant mean curvature by rolling. 


\section{Rolling construction and a generalization of Delaunay theorem}

Suppose $\Gamma$ is a plane curve given by polar coordinate graph $r=r(\theta)$, e.g., an ellipse can be expressed as the polar coordinate graph of $r=$ $e p /(1-e \cos \theta)$ with origin at one of its focus. If one rolls $\Gamma$ on the $x$-axis, then the trace of the origin of the polar coordinate system attached to $\Gamma$ plots another curve $\Omega$. Let us first investigate the analytical relationship between $\Gamma$ and $\Omega$.

As indicated in Fig. 2, $\xi$ is the arc length of $\Gamma$ starting from $Q_{0}$. Let $s$ be arc length of $\Gamma$ starting from $P_{0}, \phi$ be the angle of the tangent vector of $\Omega$ at $P$, and $\bar{\phi}$ be the angle of $\overrightarrow{Q P}$.

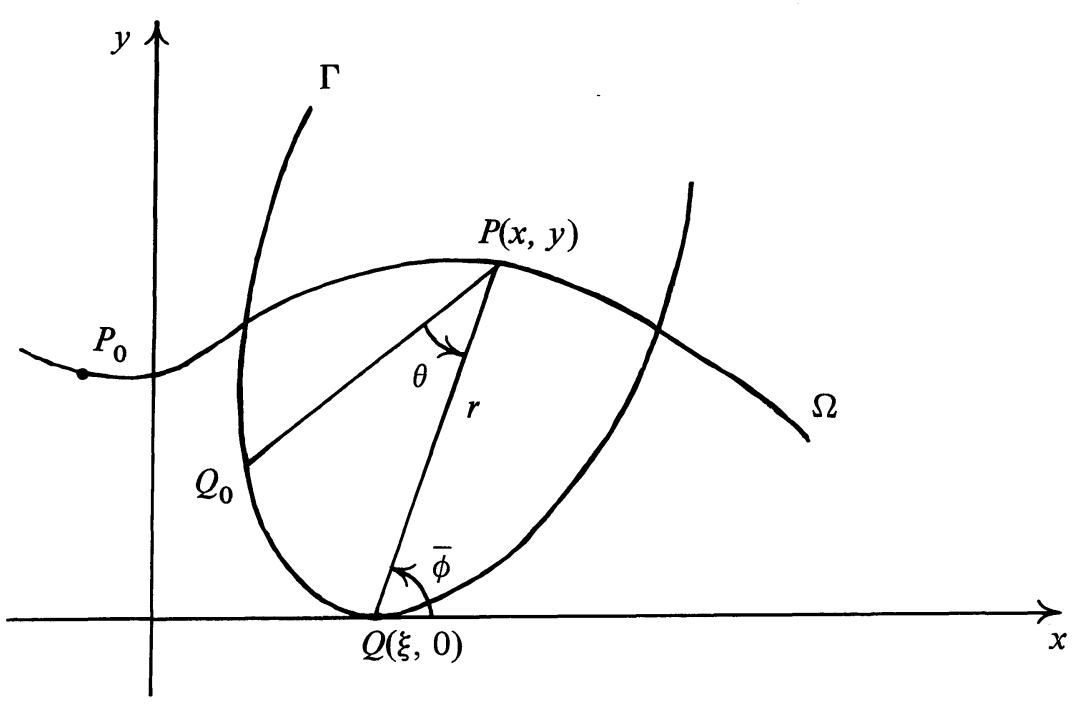

FIG. 2

Then it is easy to see the following relationships

$$
x=\xi+r \cos \bar{\phi}, \quad y=r \sin \bar{\phi}, \quad d r=-\cos \bar{\phi} \cdot d \xi
$$

It follows easily from (6) that

$$
\cos \bar{\phi} \cdot d x+\sin \bar{\phi} \cdot d y=\cos \bar{\phi} \cdot d \xi+d r=0 .
$$

Hence $P Q$ is normal to $\Omega$, and $\phi=\bar{\phi}-\pi / 2$. By differentiating with respect to the arc length $s$ of $\Omega$, one obtains

$$
\begin{aligned}
& r=\frac{y}{\cos \phi}, \\
& d r=\frac{1}{\cos \phi} d y+\frac{y}{\cos ^{2} \phi} \sin \phi d \phi=\tan \phi(1+r \phi) d s ;
\end{aligned}
$$




$$
\begin{aligned}
& \xi=x+r \sin \phi \\
& d \xi=d x+\sin \phi \cdot d r+r \cos \phi d \phi=\sec \phi(1+r \dot{\phi}) d s .
\end{aligned}
$$

Therefore it follows that

$$
d \theta= \pm \frac{1}{r} \sqrt{d \xi^{2}-d r^{2}}= \pm \frac{1}{r}(1+r \dot{\phi}) d s .
$$

(By the choice of directions of the curves $\Gamma$ and $U$ (cf. Fig. 2), we should take the + sign in (10).)

Proposition 2. Suppose $\Omega$ is a $C^{2}$-curve given by $y=f(x)>0$. If the center of curvature of $\Omega$ never lies on the $x$-axis, then there exists a unique polar coordinate graph $\Gamma$ such that $\Omega$ is the trace of the origin by rolling $\Gamma$ on the $x$-axis.

Proof. Under the assumption of the proposition, one always has $1+r \dot{\phi}$ $\neq 0$. Therefore it never changes its sign. Choose a starting point $\left(x_{0}, y_{0}\right)$ and assign the corresponding values of $s=0, \theta=0, r=r_{0}=y_{0} / \cos \phi_{0}$. Then

$$
\theta=\theta(s)=\int_{0}^{s} \frac{1}{r}(1+r \dot{\phi}) d s
$$

is clearly a strictly monotonic function of $s$. Hence one may solve for $s$ in terms of $\theta$ and substitute it into $r=y / \cos \phi=r(s)$ to obtain the polar coordinate equation $r=r(\theta)$. It is rather straightforward to verify that if one rolls the plane curve $\Gamma$ (defined by the above polar coordinate graph $r=$ $r(\theta))$ on the $x$-axis, then the trace of the origin of its attached polar coordinate system is exactly the given $\Omega$. q.e.d.

Next let us assume that $\Omega$ is a solution curve of (2).

Lemma 1. Suppose $\Omega$ is a solution curve of (2). If there exists a point $P$ on $\Omega$ whose center of curvature lies on the $x$-axis or $\Omega$ intersect with the $x$-axis, then $\Omega$ is a circular arc with its center on the $x$-axis and $(n-1) /|h|$ as its radius.

Proof. The first case follows from the uniqueness of solution of (2) at a regular point, and the second case from the analyticity as well as the uniqueness of solutions of (2) at a boundary singular point (cf. 4). q.e.d.

In view of the above lemma, from now on we shall assume that $\Omega$ is a solution curve of (2) different from a circular arc. Therefore it follows from Proposition 2 that there exists a unique polar coordinate graph $\Gamma$ given by $r=r(\theta)$.

Theorem 1. Suppose $\Omega$ is not a circular arc. Then $\Omega$ satisfies (2) if and only if its corresponding $\Gamma$ satisfies

$$
\frac{d^{2}}{d \theta^{2}}(\ln r)=\left[1+\left(\frac{d}{d \theta} \ln r\right)^{2}\right] \frac{(n-2)+h r}{(n-1)+h r}
$$


Proof. It follows from (8) and (10) that

$$
\frac{d r}{d \theta}=r \cdot \tan \phi, \quad \text { or } \frac{d}{d \theta}(\ln r)=\tan \phi .
$$

Observe that $\dot{x} \ddot{y}-\dot{y} \ddot{x}=\dot{\phi}$ and $\dot{x} / y=\cos \phi / y=1 / r$. Then it follows from (2) and (10) that

$$
\frac{d \phi}{d \theta}=\frac{r \dot{\phi}}{1+r \dot{\phi}}=\frac{(n-2)+r h}{(n-1)+r h} .
$$

Differentiating (13), one has

$$
\frac{d^{2}}{d \theta^{2}}(\ln r)=\left(1+\tan ^{2} \phi\right) \frac{d \phi}{d \theta},
$$

which, together with (13) and (14), gives the differential equation (12) satisfied by $\Gamma$.

Conversely, suppose $\Gamma$ is a polar coordinate graph given by a solution $r=r(\theta)$ of (12), and $\Omega$ is the trace of the origin by rolling $\Gamma$ on the $x$-axis. We shall verify that $\Omega$ is the graph of $y=f(x)$ which satisfies (2). As shown in Fig. 2, $\phi=\bar{\phi}-\pi / 2$, hence

$$
\tan \phi=-\cot \bar{\phi}=\frac{1}{r} \frac{d r}{d \theta}=\frac{d}{d \theta}(\ln r) .
$$

Again it follows from (10) that $d \phi / d \theta=r \dot{\phi} /(1+r \dot{\phi})$. Therefore by differentiating the above equation and making use of (12), one has

$$
\frac{r \dot{\phi}}{1+r \dot{\phi}}=\frac{(n-2)+h r}{(n-1)+h r} \text { or } r \dot{\phi}=(n-2)+h r .
$$

This proves that $\Omega$ is a solution of $\dot{\phi}-(n-2) / r=h$ or $\dot{\phi}=(n-2) \dot{x} / y=$ $h$. q.e.d.

Combining Proposition 2 with Theorem 1, one demonstrates that it is always possible to use the rolling construction to transform the problem of solving (2) to the problem of solving (12). As it was pointed out in $\$ 1,(2)$ can, in fact, already be solved explicitly in terms of hyperelliptic functions. Hence it is rather natural to seek explicit solutions of (12) and then try to analyze whether such a geometric transformation actually simplifies the original problem or at least improves our understanding of its solutions. In order to solve (12) explicitly, it is natural to introduce the following auxiliary variables, namely,

$$
u=\ln r, \quad v=\frac{d r}{d \theta}=\frac{1}{r} \frac{d r}{d \theta}
$$


Then

$$
\frac{d^{2}}{d \theta^{2}} \ln r=\frac{d v}{d \theta}=\frac{d u}{d \theta} \frac{d v}{d u}=v \frac{d v}{d u}
$$

and hence (12) becomes

$$
v \frac{d v}{d u}=\left(1+v^{2}\right) \frac{(n-2)+h e^{u}}{(n-1)+h e^{u}}
$$

or

$$
\frac{d}{d u} \ln \left(1+v^{2}\right)=2 \frac{(n-2)+h e^{u}}{(n-1)+h e^{u}} .
$$

Integrating both sides of (15), one has

$$
\ln \left(1+v^{2}\right)=\frac{2(n-2)}{(n-1)} u+\frac{2}{(n-1)} \ln \left|n-1+h e^{u}\right|+\alpha_{1}
$$

That is,

$$
\left(1+v^{2}\right)=\alpha e^{2(n-2) /(n-1)} u\left|n-1+h e^{u}\right|^{2 /(n-1)}, \quad \alpha=e^{\alpha_{1}}
$$

or

$$
\frac{1}{r} \frac{d r}{d \theta}=v= \pm\left[\alpha r^{2(n-2) /(n-1)}|n-1+h r|^{2 /(n-1)}-1\right]^{-1 / 2} .
$$

Let $w=1 / r$. Then

$$
\frac{d w}{d \theta}=\mp\left\{\alpha|(n-1) w+h|^{2 /(n-1)}-w^{2}\right\}^{1 / 2}=\mp b(w)^{1 / 2},
$$

where $b(w)=\alpha|(n-1) w+h|^{2 /(n-1)}-w^{2}$. Hence

$$
\theta=\mp \int b(w)^{-1 / 2} d w=\mp A(w)=c_{1} .
$$

Lemma 2. Let $b_{1}(w)=\left\{\alpha[(n-1) w+h]^{2 /(n-1)}-w^{2}\right\},-h /(n-1) \leqslant w$ $\leqslant \infty, n \geqslant 3, \alpha>0$. Then $b_{1}(w)$ satisfies the following properties:

(i) If $h<0$ and $\alpha \leqslant(-h / n-2)^{2(n-2) /(n-1)}$, then $b_{1}(w)<0$.

(ii) If $h \geqslant 0$, or $h<0$ and $\alpha>(-h / n-2)^{2(n-2) /(n-1)}$, then $b_{1}(w)$ has exactly two simple roots $w_{1}$ and $w_{2}$, say $w_{1}<w_{2}$, and $b_{1}(w) \geqslant 0$ for $w \in$ $\left[w_{1}, w_{2}\right]$.

(iii) $b_{1}(w) \geqslant-\left(w-w_{1}\right)\left(w-w_{2}\right), w \in\left[w_{1}, w_{2}\right]$, and

$$
\begin{aligned}
& w_{1}=0 \quad \text { if } h=0, \\
& \frac{-h}{n-1}<w_{1}<0<w_{2}, \quad \text { if } h>0, \\
& \frac{-h}{(n-1)}<w_{1}<\frac{-h}{(n-2)}<w_{2}, \quad \text { if } h<0 .
\end{aligned}
$$


Proof. $\quad b_{1}^{\prime}(w)=2 \alpha[(n-1) w+h]^{(3-n) /(n-1)}-2 w$,

$$
b_{1}^{\prime \prime}(w)=2(3-n) \alpha[(n-1) \cdot w+h]^{(4-2 n) /(n-1)}-2, \quad n \geqslant 3 .
$$

Therefore $b_{1}^{\prime \prime}(w) \leqslant-2$, and hence $b_{1}^{\prime}(w)$ has at most one simple root. From the above facts, it is easy to verify that $b_{1}(w)$ has all the above three properties.

Lemma $2^{\prime}$. Let $b_{2}(w)=\left\{\alpha[-(n-1) w-h]^{2 /(n-1)}-w^{2}\right\}, h<0,-\infty<$ $w \leqslant-h /(n-1)$. Then $b_{2}(w)$ satisfies the following properties:

(i) $b_{2}(w)$ has two simple roots $w_{1}, w_{2}$ and $w_{1}<0<w_{2}<-h /(n-1)$.

(ii) $w_{1}<-w_{2}$ and $b_{2}(w) \geqslant 0$ if and only if $w_{1} \leqslant w \leqslant w_{2}$; moreover, $b_{2}(w)$ $\geqslant-\left(w-w_{1}\right)\left(w-w_{2}\right)$ for $w \in\left[w_{1}, w_{2}\right]$.

Proof. Again one has $b_{2}^{\prime \prime}(w) \leqslant-2$, and hence $b_{2}^{\prime}(w)$ has at most one simple root. Moreover, it is clear that $b_{2}(-h /(n-1))<0$ and $b_{2}(0)>0$. Therefore it is straightforward to verify that $b_{2}(w)$ has properties (i) and (ii). q.e.d.

Based on the above properties of $b_{i}(w), i=1,2$, one may define the antiderivatives $A_{i}(w)$ of $b_{i}(w)^{-1 / 2}$ more precisely as follows:

$$
\begin{gathered}
A_{1}(w)=\int_{\max \left(0, w_{1}\right)}^{w}\left\{\alpha[(n-1) t+h]^{2 /(n-1)}-t^{2}\right\}^{-1 / 2} d t, \\
w \in\left[\max \left(0, w_{1}\right), w_{2}\right], \\
A_{2}(w)=\int_{0}^{w}\left\{\alpha[-(n-1) t-h]^{2 /(n-1)}-t^{2}\right\}^{-1 / 2} d t, \quad w \in\left[0, w_{2}\right] .
\end{gathered}
$$

Summarizing the discussion of this section, we state the generalization of Delaunay theorem as follows.

Theorem 2. Suppose $N$ is a generalized rotational surface of $(S O(n-$ 1), $\left.\rho_{n-1}+1, \mathbf{R}^{n}\right)$-type and of constant mean curvature $h$, and $\Omega=N / G \subset$ $\mathbf{R}^{n} / G \simeq \mathbf{R}^{2} / \mathbf{Z}_{2}$ is its image curve which can be given as a graph of $y=f(x) \geqslant$ 0 in the upper half plane. Then one has the following possibilities:

I. Case $h<0$.

(i) $\Omega$ is a circular arc with its center on the $x$-axis and $(n-1) /(-h)$ as its radius,

(ii) $\Omega$ is given by $y=(n-2) /(-h)$,

(iii) $y / \cos \phi<(n-1) /(-h)$, and $\Omega$ is obtained by rolling a uniquely determined curve $\Gamma$ whose polar coordinate equation is given by $\theta=$ $\pm A_{1}(1 / r)+c$.

(iv) $y / \cos \phi>(n-1) /(-h)$, and $\Omega$ is obtained by rolling a uniquely determined curve $\Gamma$ whose polar coordinate equation is given by $\theta= \pm A_{2}(1 / r)$ $+c$. 
II. Case $h \geqslant 0$. $\Omega$ is obtained by rolling a uniquely determined curve $\Gamma$ whose polar coordinate equation is given by $\theta= \pm A_{1}(1 / r)+c$.

We shall make use of the above theorem to investigate various properties of $\Omega$ via that of $\Gamma$ in $\S 3$.

\section{Properties of generalized rotation hypersurfaces of constant mean curvature of $\left(S O(n-1), \mathbf{R}^{n}\right)$-type}

In this section we shall apply the rolling construction of $\$ 2$ to investigate some interesting properties of generalized rotational hypersurfaces of constant mean curvature of $\left(S O(n-1), \mathbf{R}^{n}\right)$-type. According to Theorems 1 and 2 , the rolling construction enable us to transfer the equation of the generating curve $\Omega$, namely (2), to that of the polar coordinate function of the rolling curve $\Gamma$, namely (12), whose solutions can be explicitly expressed in terms of the following integral:

$$
\theta= \pm \int b_{i}(w)^{-1 / 2} d w, \quad i=1,2, w=\frac{1}{r}
$$

Therefore it is easy to use the above integral expression to study some interesting properties of $\Omega$ such as periodicity via the rolling construction. In the classical case of $n=3$

$$
b_{1}(w)=\alpha \cdot(2 w+h)-w^{2}, \quad b_{2}(w)=-\left[\alpha(2 w+h)+w^{2}\right]
$$

are simply quadratic polynomials of $w$. Therefore it is straightforward to compute $\theta= \pm \int b_{i}(w) d w$ in terms of trigonometric functions. Explicit computation will show that

$$
\frac{1}{r}=w=a+b \cos (\theta+c), \quad(a, b, c: \text { suitable constants }),
$$

which is exactly the polar coordinate function of a conic section with a focus as its origin. This gives us the classical Delaunay theorem.

In the general case of $n \geqslant 4$, Lemmas 2 and $2^{\prime}$ provide those essential properties of the functions $b_{i}(w), i=1,2$, for our investigation of the integral $\theta= \pm \int b_{i}(w)^{-1 / 2} d w$. We shall divide our discussion as follows.

\subsection{Periodic solutions}

In the classical case of $n=3$, if $\Gamma$ is an ellipse then $\Omega$ is periodic, namely, $\Omega$ is given by a periodic function $y=f(x), f(x+T) \equiv f(x)$. In the general case, $\Omega$ is periodic if and only if $\Gamma$ is periodic, and hence a necessary condition for $\Omega$ to be periodic will be that $r=r(\theta)$ is positive and bounded. In view of 
Theorem 2 and Lemmas $2,2^{\prime}$, the above necessary condition implies that $\theta= \pm \int b_{1}(w)^{-1 / 2} d w$, and $h<0, \alpha>[-h /(n-2)]^{2(n-2) /(n-1)}$. In fact, it is not difficult to deduce the following theorem from Theorem 2 and Lemma 2 by straightforward computation.

Theorem 3. $\Omega$ is periodic if and only if $\Gamma$ is given by the following polar coordinate function, namely, $\theta= \pm \int b_{1}(w)^{-1 / 2} d w$ with $w=1 / r, b_{1}(w)=$ $\left\{\alpha[(n-1) w+h]^{2 /(n-1)}-w^{2}\right\}$ and $h<0, \alpha>[-h /(n-2)]^{2(n-2) /(n-1)}$. By Lemma $2, b_{1}(w)$ has two simple roots $w_{1}, w_{2},-h /(n-1)<w_{1}<-h /(n-2)$ $<w_{2}$. Then, up to a translation along the $x$-axis, $\Omega$ is given by $y=f(x)$ satisfying the following properties:

(i) $f(x)$ is a periodic function with its period $T<-2 h(1+\pi) /(n-1)$,

(ii) $f(0)=1 / w_{1}<-(n-1) / h, f(T / 2)=1 / w_{2}$, and $f(x)$ is symmetric with respect to $x=T / 2$,

(iii) $f(x)$ is strictly decreasing between 0 and $T / 2$, and strictly increasing between $T / 2$ and $T$.

Proof. Theorem 2 reduces the verification of above theorem to the corresponding properties of $\Gamma$. Let $\theta=\int_{w_{1}}^{w} b_{1}(t)^{-1 / 2} d t$. Then the properties of $b_{1}(t)$ listed in Lemma 2 and simple estimates will show that

(i) $w$ is a strictly increasing function of $\theta$ between 0 and $\theta^{*}=$ $\int_{w_{1}}^{w_{2}} b_{1}(t)^{-1 / 2} d t$

(ii) $d w / d \theta=0$ at both $\theta=0$ and $\theta=\theta^{*}$.

Therefore it follows from the uniqueness of (12) that one may analytically continue the solution $w=w(\theta)$ of (12) simply by reflection with respect to $\boldsymbol{\theta}=k \boldsymbol{\theta}^{*}$, namely,

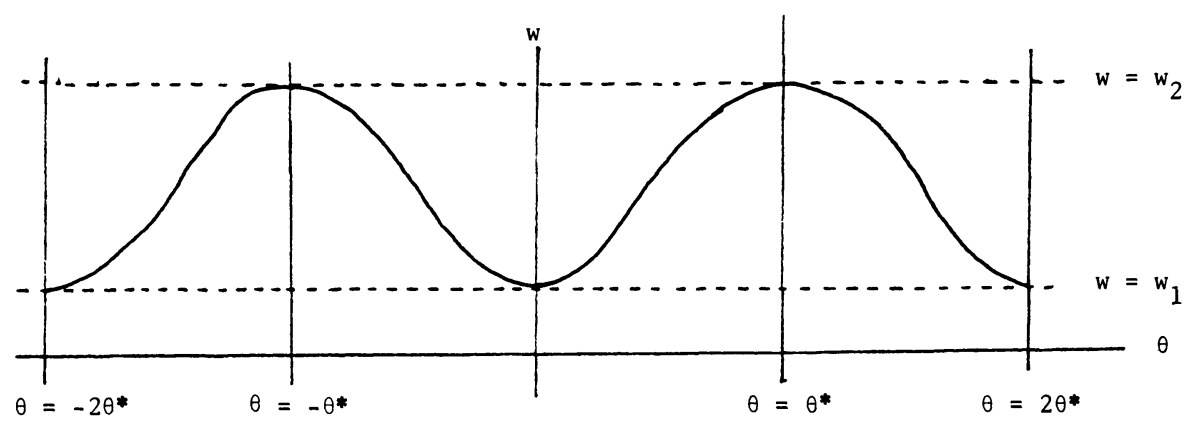

FIG. 3

Hence $\Gamma$ is a periodic curve of period $2 \theta^{*}$, and consequently $\Omega$ is also periodic whose period $T$ is equal to the arc length of $\Gamma$ between $\theta=0$ and $\theta=2 \theta^{*}$. 
It is easy to use the properties $b_{1}(w) \geqslant-\left(w-w_{1}\right)\left(w-w_{2}\right)$ and $w_{1}>$ $-(n-1) / h$ to estimate that

$$
T=2 \int_{0}^{\theta^{*}}\left[r^{2}+\left(\frac{d r}{d \theta}\right)^{2}\right]^{1 / 2} d \theta<\frac{-2 h}{(n-1)}(1+\pi) .
$$

COROLlARY. As a corollary of Theorem 3 and a theorem of Ruh and Vilms [6], the Gauss map of the generalized rotational hypersurface of $\Omega$ in $\mathbf{R}^{n}$ is a periodic harmonic map of $\mathbf{R}^{1} \times S^{n-2} \rightarrow S^{n-1}$, and hence its quotient is a harmonic map of degree zero of $S^{1} \times S^{n-2} \rightarrow S^{n-1}$.

\subsection{Generalized rotational minimal hypersurfaces in $\mathbf{R}^{\boldsymbol{n}}$}

In the classical case of $n=3$, the constant mean curvature $h=0$ if and only if $\Gamma$ is a parabola. The generating curve $\Omega$ is given by $y=$ $\frac{1}{c} \cosh \left(c\left(x-x_{0}\right)\right)$. In the case $n \geqslant 4$, it is to specialize the explicit formula of (5) to obtain the following:

$$
x=\frac{1}{c} g(c \cdot y)+x_{0}, \quad g(u)=\int_{1}^{u} \frac{d t}{\sqrt{t^{2 n-4}-1}} .
$$

Therefore the properties of $\Omega$ can easily be investigated directly from the above formula (without going through the following construction).

\subsection{The hyperbolic type}

Suppose $n=3$, and $\Gamma$ is a hyperbola. Then we have the following two different possibilities, namely,

(i) if one rolls a branch of hyperbola on the upper side of the $x$-axis, then the focus inside of the branch traces a curve $\Omega$ whose rational surface has constant mean curvature $h>0$.

(ii) if one rolls a branch of hyperbola on the lower side of the $x$-axis, then the focus outside of the branch traces a curve $\Omega$ whose rotational surface has constant mean curvature $h<0$.

Correspondingly, one also has the following two possibilities for the general case of $n \geqslant 4$, namely,

(i) $h>0$. In this case $b_{1}(w)=\left\{\alpha[(n-1) w+h]^{2 /(n-1)}-w^{2}\right\}$ has exactly two simple roots $w_{1}, w_{2}$ and $(-h) /(n-1)<w_{1}<0<w_{2}$. The curve $\Gamma$ is given by the polar coordinate relationship:

$$
\theta= \pm \int_{0}^{w} b_{1}(t)^{-1 / 2} d t+\theta_{0}
$$


Let $\theta^{*}=\int_{0}^{w_{2}} b_{1}(t)^{-1 / 2}$. Then it is not difficult to show that $\Gamma$ is a curve of the following shape:

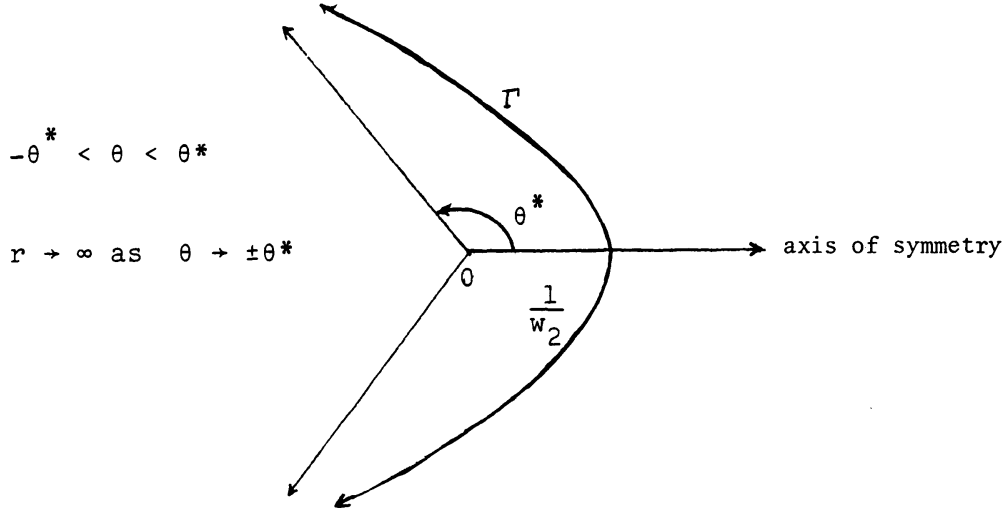

Fig. 4

$\Omega$ is obtained by rolling the above $\Gamma$ on the upper side of the $x$-axis.

(ii) $h<0$ and $\Gamma$ is given by the following polar coordinate relationship:

$$
\begin{gathered}
\theta= \pm \int_{0}^{w} b_{2}(t)^{-1 / 2} d t+\theta_{0} \\
b_{2}(t)=\left\{\alpha[-(n-1) t-h]^{2 /(n-2)}-t^{2}\right\} .
\end{gathered}
$$

By Lemma $2^{\prime}, b_{2}(w)$ has two simple roots $w_{1}, w_{2}, w_{1}<0<w_{2}<$ $(-h) /(n-1)$. Let $\theta^{*}=\int_{0}^{w_{2}} b_{2}(t)^{-1 / 2} d t$. Again it is not difficult to see that $\Gamma$ is then a curve of the following shape:

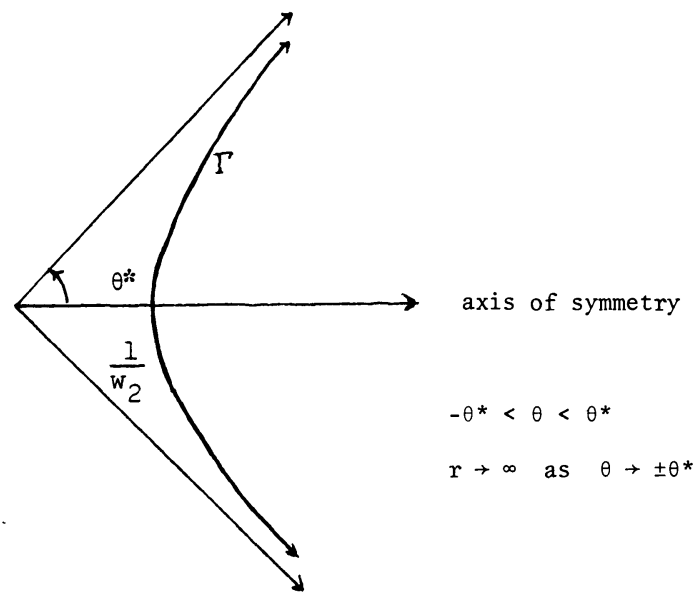

Fig. 5

$\Omega$ is obtained by rolling $\Gamma$ on the lower side of the $x$-axis. 
It is rather straightforward to use the rolling construction and the integral expression of $\Gamma$ to investigate further properties of $\Omega$ in the above two cases of hyperbolic type. We state some of their basis properties as follows without proof.

Theorem 3'. The generating curve $\Omega$ of hyperbolic type has the following properties:

(a) If $h>0$, then, up to a translation along the $x$-axis, $\Omega$ is given by $y=f(x)$ satisfying the following properties:

(i) $f(x)$ is a convex function symmetric with respect to $x=0$,

(ii) $\Omega$ is a finite length open arc defined over $(-\beta, \beta)$ with $\beta<1 / h$, $\lim _{x \rightarrow \beta} f^{\prime}(x)=+\infty$ and $\lim _{x \rightarrow \beta} f(x)=\gamma<+\infty$.

(b) If $h^{\prime}<0$, then, up to a translation along the $x$-axis, $\Omega$ is given by $y=f(x)$ satisfying the following properties:

(i) $f(x)$ is defined over $\left[-\beta^{\prime}, \beta^{\prime}\right], \beta^{\prime}<(n-1) /\left|h^{\prime}\right|$ and symmetric with respect to $x=0$,

(ii) $f(x)$ is strictly increasing over $\left(-\beta^{\prime}, 0\right)$ and strictly decreasing over $\left(0, \beta^{\prime}\right), f^{\prime \prime}(x)>0, \lim _{x \rightarrow \beta^{\prime}} f^{\prime}(x)=-\infty, \lim _{x \rightarrow \beta^{\prime}} f(x)=\gamma^{\prime}<+\infty$.
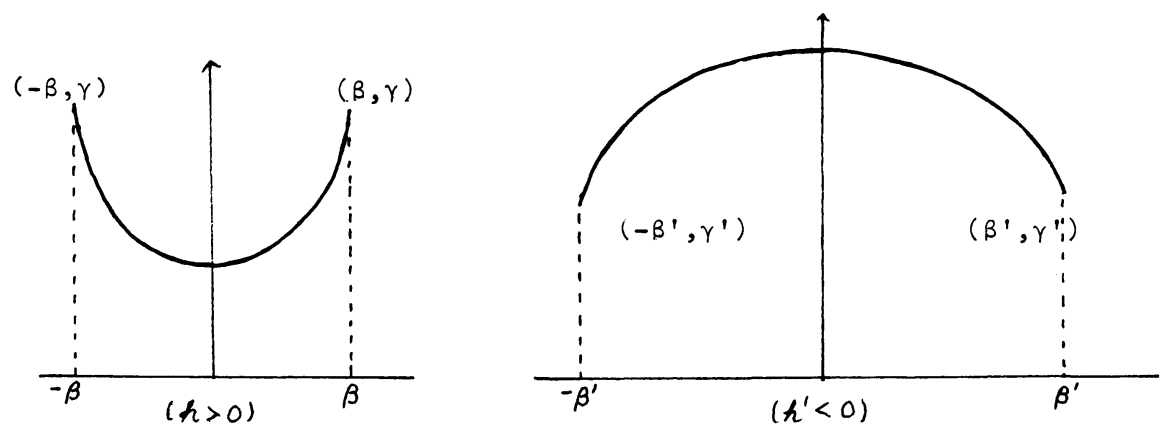

FIG. 6

(c) By suitably adjusting the value of $\alpha$, one may realize an arbitrary positive value for $\gamma$ or $\gamma^{\prime}$. Therefore it is easy to piece the above two kinds of generating curves with $h^{\prime}=-h$ and $\gamma^{\prime}=\gamma$ by translation and uniqueness. In this way, one obtains periodic solutions of the following type whose Gauss map provides harmonic maps of $S^{1} \times S^{n-2} \rightarrow S^{n-1}$, which covers $S^{n-1}$ twice generically, and hence also those examples which cover $S^{n-1} 2 k$ times generically by taking longer period. 


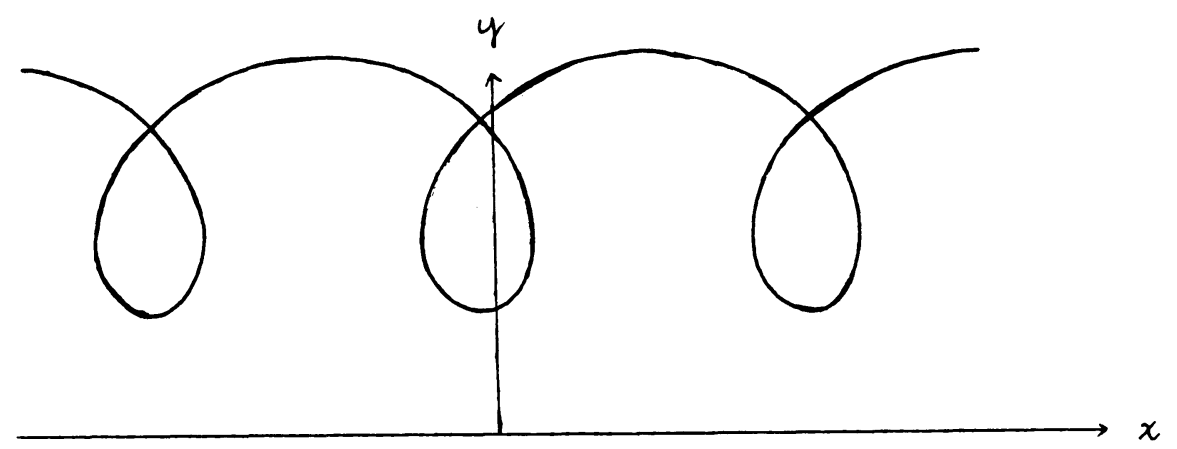

FIG. 7

\subsection{Concluding remarks}

(i) The rolling construction is a simple geometric construction which establishes a simple, rigid, analytic relationship between the cartesian expression of $\Omega$ and the polar expression of $\Gamma$. In the case that $\Omega$ is a generating curve of a generalized rotational hypersurface of constant mean curvature in $\mathbf{R}^{n}$, the polar coordinate expression of $\Gamma$ is given by a rather simple integral, namely, $\theta= \pm \int b_{i}(w) d w$. Therefore it is usually advantageous to investigate the properties of $\Omega$ via the corresponding properties of $\Gamma$.

(ii) In the case $h<0$, there are four types of solutions for the generating curve $\Omega$, namely,

(1) constant solution: $y=(n-2) /(-h)$,

(2) circular solution: $y=\left[((n-1) / h)^{2}-x^{2}\right]^{1 / 2}$,

(3) periodic solutions of elliptic type,

(4) solutions of hyperbolic type.

Geometrically, they can all be fitted into a continuous family with the constant solution as the limit of periodic solutions and the circular solution as the dividing point of periodic solutions of elliptic type and that of hyperbolic type.

(iii) In the periodic case, one constructs a family of harmonic maps of $S^{k} \times T^{l} \rightarrow S^{k+1} \times T^{-1}$. It is not difficult to check that the composition $S^{k} \times T^{l} \rightarrow S^{k+1} \times T^{l-1} \rightarrow S^{k+2} \times T^{l-2}$ is no longer harmonic. However, it is rather tempting to see if some suitable modification of the above composition will yield a harmonic map of $S^{k} \times T^{l} \rightarrow S^{k+2} \times T^{l-2}$.

\section{References}

[1] C. Delaunay, Sur la surface de révolution dont la courbure mayenne est constante, J. Math. Pures. Appl. Sér. 1, 6 (1841) 309-320.

[2] J. Eells, On the surfaces of Delaunay and their Gauss maps, to appear. 
[3] W. Y. Hsiang \& W. T. Hsiang, On the construction of exotic and/or knotted spheres of constant mean curvature in the standard sphere, to appear.

[4] W. Y. Hsiang \& B. Lawson, Minimal submanifolds of low cohomogeneity, J. Differential Geometry 5 (1971) 1-38.

[5] K. Kenmotsu, Surface of revolution with prescribed mean curvature, Tôhoku Math. J. 32 (1980) 147-153.

[6] E. A. Ruh \& J. Vilms, The tension field of the Gauss map, Trans. Amer. Math. Soc. 149 (1970) 569-573.

FudAN University, SHANGHAI

UNIVERSITY OF CALIFORNIA, BERKELEY 
